\title{
Lipopolysaccharide promotes lung fibroblast proliferation through autophagy inhibition via activation of the PI3K-Akt-mTOR pathway
}

\author{
Tingting Xie ${ }^{1} \cdot$ Qiaoyi Xu ${ }^{1} \cdot$ Hanxi Wan ${ }^{1} \cdot$ Shunpeng Xing ${ }^{1} \cdot$ Chen Shang $^{1} \cdot$ Yuan Gao ${ }^{1} \cdot$ Zhengyu He$^{1}$
}

Received: 10 December 2017 / Revised: 5 October 2018 / Accepted: 25 October 2018 / Published online: 13 February 2019

(c) United States \& Canadian Academy of Pathology 2019

\begin{abstract}
Pulmonary fibrosis is a major cause of death in patients with acute respiratory distress syndrome (ARDS). Our previous study revealed that lipopolysaccharide (LPS) challenge could lead to mouse lung fibroblast proliferation. Additionally, inhibition of autophagy in lung fibroblasts was also reported to be crucial during the process of pulmonary fibrosis. However, the correlation between proliferation and inhibition of autophagy of lung fibroblasts and the underlying mechanism remain unknown. In this study, we report that autophagy was inhibited in mouse lung fibroblasts after LPS challenge, and was accompanied by activation of the PI3K-Akt-mTOR signaling pathway. Treating mouse lung fibroblasts with LPS resulted in mTOR and Akt phosphorylation, p62 up-regulation, and significant down-regulation of autophagosomes, which could be reversed by PI3K-Akt inhibitors (Ly294002) or mTOR inhibitors (rapamycin, RAPA). Furthermore, either LPS or hydroxychloroquine (HCQ), an autophagy inhibitor, could promote mouse lung fibroblast proliferation, which could be reversed by RAPA application. The present research therefore reveals that LPS promotes lung fibroblast proliferation through autophagy inhibition via activation of the PI3K-Akt-mTOR pathway.
\end{abstract}

\section{Introduction}

Pulmonary fibrosis, which is characterized by the aberrant activation and proliferation of lung fibroblasts and the abnormal secretion of collagen, has traditionally been regarded as a late event of acute respiratory distress syndrome (ARDS). However, recent studies have suggested that lung collagen turnover increases within $24 \mathrm{~h}$ of ARDS diagnosis and that this symptom is related to poor patient prognosis [1]. Intractable pulmonary fibrosis has been reported as a major cause of death in patients with ARDS [2].

Lipopolysaccharide (LPS) is a prominent component of Gram-negative bacilli endotoxins and an important

These authors contributed equally: Tingting Xie, and Qiaoyi Xu

Yuan Gao

rj_gaoyuan@163.com

$\bowtie$ Zhengyu He

zhengyuheshsmu@163.com

1 Department of Critical Care Medicine, Renji Hospital, School of Medicine, Shanghai Jiaotong University, Shanghai, China etiological factor in ARDS and pulmonary fibrosis [3-7]. Our previous study showed that LPS induces lung fibroblast proliferation through the activation of phosphoinositide3-Kinase (PI3K)-Akt signaling pathway $[8,9]$. Recently, it was reported that autophagy is inhibited in lung fibroblasts of idiopathic pulmonary fibrosis (IPF) patients [10]. Activation of mammalian target of rapamycin (mTOR), a downstream molecule of the PI3KAkt signaling pathway, inhibits autophagy. Autophagy is a lysosome-dependent, self-digestive process by which dysfunctional cellular components and waste proteins are degraded. This process promotes cellular survival during stress, starvation or nutrient restriction by maintaining essential cellular activity and viability. A low level of autophagy was detected in the lung tissues and fibroblasts of IPF patients [11], inferring a relationship between autophagy inhibition and pulmonary fibrosis. However, the correlation between proliferation and autophagy inhibition of lung fibroblasts and the underlying mechanism remains unknown.

In this study, we explored the relationship between lung fibroblast proliferation and autophagy inhibition and to clarify the important role PI3K-Akt-mTOR pathway may play in the process. 


\section{Materials and methods}

\section{Primary cultures of mouse lung fibroblasts (MLFs)}

Lung fibroblasts were isolated from C57BL/6 mice (8 weeks old, from Shanghai SLAC Laboratory Animal Co. Ltd., China) as described in our previous study [7]. All procedures of this study were carried out in accordance with the guidelines for animal care published by the United States' National Institutes of Health (NIH) for animal care (Guide for the Care and Use of Laboratory Animals, Department of Health and Human Services, NIH Publication No. 86-23, revised 1985). All animals were kept in a pathogen-free environment and fed ad libitum. Efforts were made to minimize the number and suffering of animals used. The procedures for care and use of animals were approved by Renji Hospital, School of Medicine, Shanghai Jiaotong University (Approval No: RJ2017-11-05). Each mouse was euthanized by decapitation, and the lung tissues were promptly excised, washed with phosphate-buffered saline (PBS, HyClone, USA) and then cut into $1 \mathrm{~mm}^{3}$ pieces. The tissues were distributed evenly on the bottom of culture plates and were mixed with Dulbecco's modified Eagle's medium (DMEM, Gibco, USA) containing 10\% fetal bovine serum (FBS, Gibco). The plates were cultured at $37^{\circ} \mathrm{C}$ in a humidified $5 \% \quad \mathrm{CO}_{2}$ incubator (Thermo Scientific, USA), and the culture medium was replaced every 3 days. When the adherent cells reached $80 \%$ confluence, they were detached by exposure to $0.25 \%$ trypsin for $2 \mathrm{~min}$ and were passaged at a 1:3 dilution. After four generations, the cells presented with the typical fusiform shape, and before being used for experiments, they were identified as fibroblasts by strong vimentin-positive immunocytochemical staining (data not shown).

\section{Experimental grouping and design}

Cells in the log phase of growth were seeded into six-well plates at a concentration of $1 \times 10^{5}$ cells $/ \mathrm{mL}(2.5 \mathrm{~mL}$ in each well) and were incubated at $37{ }^{\circ} \mathrm{C}$ in a humidified $5 \% \mathrm{CO}_{2}$ incubator. The fibroblasts adhered to the bottom of the culture plates $8 \mathrm{~h}$ after inoculation. The DMEM containing $10 \%$ FBS was replaced with serum-free DMEM, and the cells were divided into the following six groups when they reached 70\% confluence: Group A (negative control), Group B (LPS stimulated), Group C (PI3K-Akt inhibited), Group D (PI3K-Akt inhibition with LPS stimulation), Group E (mTOR inhibited), and Group F (mTOR inhibition with LPS stimulation). Group A (negative control groups, Con.) cells were treated with PBS with the same volume of LPS and/or inhibitors in the following groups. Group B (LPS-stimulated groups, LPS) cells were treated with LPS. Group C (PI3K-Akt-inhibited groups, Ly294002) cells were treated with Ly294002, an inhibitor of the PI3K-Akt signaling pathway, but were not stimulated with LPS. Group D (PI3K-Akt inhibited groups with LPS stimulation, LPS + Ly294002) cells were pretreated with Ly294002 for $1 \mathrm{~h}$ prior to LPS stimulation. Group E (mTOR inhibition groups, RAPA) cells were treated with rapamycin, an inhibitor of mTOR, without LPS stimulation. Group F (mTOR inhibition groups stimulated by LPS, LPS + RAPA) cells were stimulated by LPS following pretreatment with rapamycin for $1 \mathrm{~h}$. LPS was applied with a concentration of $1 \mu \mathrm{g} / \mathrm{mL}$. Ly294002 and Rapamycin were applied with concentration of $10 \mu \mathrm{M}$ and $10 \mathrm{nM}$ according to the recommended dilutions. Our preliminary studies found that the expression of PI3K-Akt-mTOR signaling proteins showed better bands $6 \mathrm{~h}$ after LPS challenged and autophagy-associated proteins showed $30 \mathrm{~h}$ after LPS challenged. Therefore, in present study, cells were collected after 6 or $30 \mathrm{~h}$ for signaling molecules detection and autophagy-associated proteins detection respectively.

In the meantime, hydroxychloroquine (HCQ), an autophagy inhibitor was also apply to inhibit lung fibroblasts autophagy according to experimental requirements.

\section{Western blot analysis}

The protein levels of LC3 and p62 and the phosphorylation levels of Akt and mTOR in each group were detected by Western blots. After stimulation with LPS for 6 or $30 \mathrm{~h}$, cells with or without inhibitors were harvested and then were lysed on ice for $15 \mathrm{~min}$ with $1 \mathrm{x}$ radioimmunoprecipitation assay (RIPA) lysis buffer (Beyotime, China) containing phenyl methyl sulfonyl fluoride (PMSF), protease inhibitor cocktail and phosphatase inhibitor cocktail (KangChen, China). Cell debris was collected and was centrifuged at $15,000 \times g$ for $10 \mathrm{~min}$ at $4{ }^{\circ} \mathrm{C}$. Then, the protein-containing supernatants were collected, and the protein concentration was determined by using of the bicinchoninic acid (BCA) protein assay kit (\#23225, Pierce, USA). SDS-polyacrylamide gel electrophoresis (PAGE) was performed; then, the separated proteins were transferred onto polyvinyl fluoride membranes (Milipore, Germany). These membranes were incubated with the appropriate primary and secondary antibodies. Protein bands were detected by using an ECL Plus Western blotting system kit (Yeason, China). The primary antibodies used in our study were as follows: rabbit anti-LC3 I/II (L7453, Sigma, USA), rabbit anti-p62/SQSTM1 (\#5114, Cell Signaling Technology, USA), rabbit anti-Akt (\#4691, Cell Signaling Technology, USA), rabbit anti-p-Akt (\#4060, Cell Signaling Technology, USA), rabbit anti-mTOR (\#2983, Cell Signaling Technology, USA), rabbit anti-p-mTOR (\#2971, Cell Signaling Technology, USA) and rabbit anti- $\beta$-actin (\#8457, Cell Signaling Technology, USA). All primary 
antibodies were used at a 1:1000 working dilution. HRPlabeled goat-anti-rabbit IgG (A0208, Beyotime, China) antibody was used as the secondary antibody (1:1000 dilution). Immunoreactive bands were visualized by ChemiDoc $^{\text {TM }}$ XRS + Molecular Imager (Bio-Rad, CA, USA) with Image Lab software (Bio-Rad, CA, USA) and were quantified with ImageJ software (v 1.46r, National Institute of Mental Health, Bethesda, USA). Protein expression was normalized to $\beta$-actin.

\section{Immunofluorescence}

The protein levels of p62 were also detected by immunofluorescence. Before cells were seeded, sterile coverslips were placed at the bottom of 12-well plates. Cells were collected after a 30- $\mathrm{h}$ incubation with LPS with/without inhibitors. Coverslips covered with monolayer fibroblasts were rinsed several times with ice-cold PBS. Then, the cells were fixed with $4 \%$ paraformaldehyde (Nanxi Biotech, China) for $10 \mathrm{~min}$ and were incubated with $0.5 \%$ Triton X-100 (Biosharp, China) in PBS for another $10 \mathrm{~min}$ to permeabilize the cells. For immunofluorescent staining, the coverslips were rinsed three times in PBS, followed by blocking in $1 \%$ bovine serum albumin (BSA, Beyotime, China) with PBS for $1 \mathrm{~h}$. The cells were then incubated at $4{ }^{\circ} \mathrm{C}$ overnight in rabbit anti-p62 primary antibody (\#ab56416, Abcam, USA) at a 1:50 working dilution. The coverslips were rinsed with PBS for $30 \mathrm{~min}$ and then were incubated in a 1:500 dilution of Alexa Fluor 488-labeled goat-anti-mouse secondary antibody (A0428, Beyotime, China) for $2 \mathrm{~h}$ at room temperature. Finally, after being rinsed several times with PBS, the coverslips were inverted on slides smeared with mounting medium and were mounted on a fluorescence microscope (D-35578, Leica Microsystems CMS, Germany) to visualize p62. All experiments were conducted independently at least 3 times. Green particles present in the cytoplasm were considered positive signals. The positive cells and total cells were separately counted in the overlapped double-stained images and analyzed. Five high power fields were randomly selected in each specimen.

\section{Transmission electron microscopy}

The formation of autophagosomes was visualized by transmission electron microscopy (TEM). Cells were collected after a 30-h incubation and were centrifuged. After centrifugation, the supernatant was discarded, and the pellet was fixed with $2.5 \%$ glutaraldehyde (G5882, MSDS, Sigma) in PBS at $4{ }^{\circ} \mathrm{C}$ overnight. After several washes in PBS, the specimen was post-fixed with $1 \%$ osmic acid (20816-12-0, Sinopharm, China) in PBS for $1 \mathrm{~h}$ and then was washed three times in PBS. Next, the specimen was stained with $2 \%$ uranyl acetate for $30 \mathrm{~min}$, was dehydrated in a graded ethanol series (30 50, 70, 90 and 100\%) for approximately 15 to $20 \mathrm{~min}$ at each step and finally was dehydrated in absolute acetone for $20 \mathrm{~min}$. The specimen was placed in a 1:1 mixture of absolute acetone and resin for $2 \mathrm{~h}$ and then was embedded with epoxy resin (EPON 812, SPI, 02660-AB, Shell Chemicals, Netherlands) for another $2 \mathrm{~h}$. To harden, the specimen was heated in an oven at $37^{\circ} \mathrm{C}$ for $24 \mathrm{~h}, 45^{\circ} \mathrm{C}$ for $24 \mathrm{~h}$, and $60^{\circ} \mathrm{C}$ for $48 \mathrm{~h}$. Ultrathin sections were prepared, followed by embedding and sectioning with an ultramicrotome (Leica EM UC7, Germany). Finally, the specimen sections were stained with uranyl acetate and alkaline lead citrate for $15 \mathrm{~min}$ and were observed under a transmission electron microscope (TECNAI 10, FEI, USA).

\section{Cell counting Kit-8 assay}

Cell proliferation was analyzed by Cell Counting Kit- 8 assay (CCK8, Dojindo, Japan). MLFs were seeded in 96well plates at a density of $4 \times 10^{3}$ cells/well and allowed to adhere for $24 \mathrm{~h}$ prior to supplementation with HCQ (Selleck Chemicals, Houston, TX, USA), LPS or rapamycin. After 6, $24,48,72 \mathrm{~h}$, the media were removed and cells were treated with $10 \%$ CCK-8 in new media for $2 \mathrm{~h}$ at $37^{\circ} \mathrm{C}$. Absorbance at $450 \mathrm{~nm}$ was measured using a spectrophotometer (Berthold Technologies, Germany). Every experiment was performed in eight parallel wells, and the mean optical density (OD) values were measured.

\section{Statistical analysis}

Data are presented as the mean \pm standard deviation (SD). The results of the expression of p62, LC3-II/I ratio, p-Akt/ Akt ratio and p-mTOR/mTOR ratio that are presented first in the Results section were analyzed by paired Student's $t$-test. All other results were analyzed by ANOVA, followed by Tukey's method. A two-sided $P$ value less than 0.05 was considered statistically significant.

\section{Results}

\section{LPS inhibits autophagy in MLFs}

First, we verified the role of LPS in autophagy in MLFs. As shown in Fig. 1a, LPS stimulation suppresses the expression of LC3-II and leads to the accumulation of p62, an autophagosome cargo protein that is degraded during autophagic flux (Fig. 1a, b). The LC3-II/I ratio declined significantly after the stimulation with LPS for $30 \mathrm{~h}$. These results indicate that LPS inhibits autophagy in MLFs. Furthermore, p62 expression increased in MLFs following the 
Fig. 1 LPS inhibits autophagy in mouse lung fibroblasts. Total cellular protein was collected from lung fibroblasts treated with $1 \mu \mathrm{g} / \mathrm{mL}$ LPS for up to 30 $\mathrm{h}$, followed by p62 and LC3 protein expression detection by Western blotting $(\mathbf{a}, \mathbf{b}) . * * p<$ 0.01 vs. Control group; *** $p<$ 0.001 vs. Control group. Columns represent mean values and error bars represent SD. Blots are representative of three independent experiments. The MLFs were stimulated with $1 \mu \mathrm{g} / \mathrm{mL}$ LPS for up to $30 \mathrm{~h}$; then, p62 expression was detected by immunofluorescence (c) and the ratio of positive-cell number to total cells were statistically analyzed in the right panel $(n=5)$. $* * p<0.01$ vs.

Control group. The autophagosomes were detected by TEM $(\mathbf{d})$ a

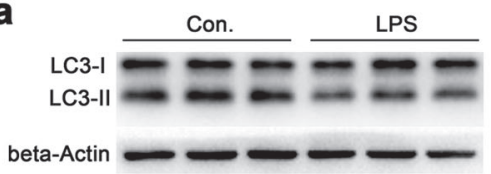

b
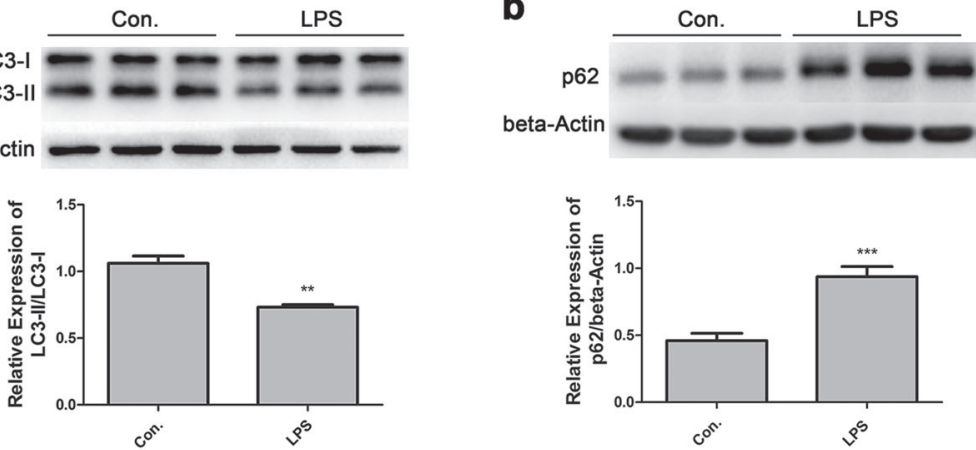

C DAPI-Nucleus Alexa Fluor 488-p62 Merged
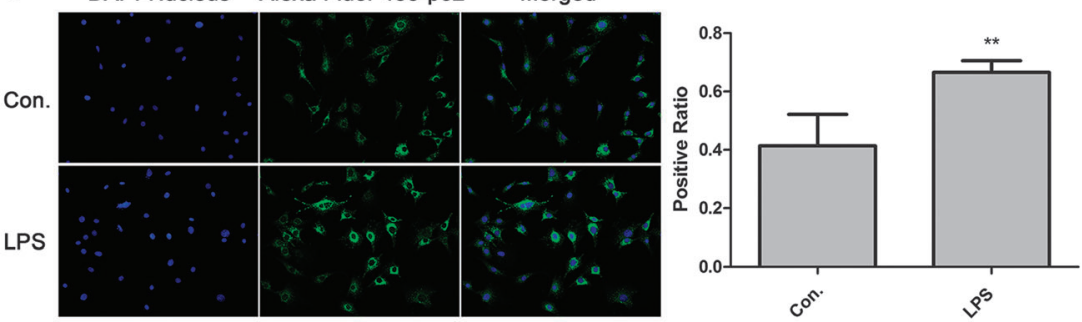

d

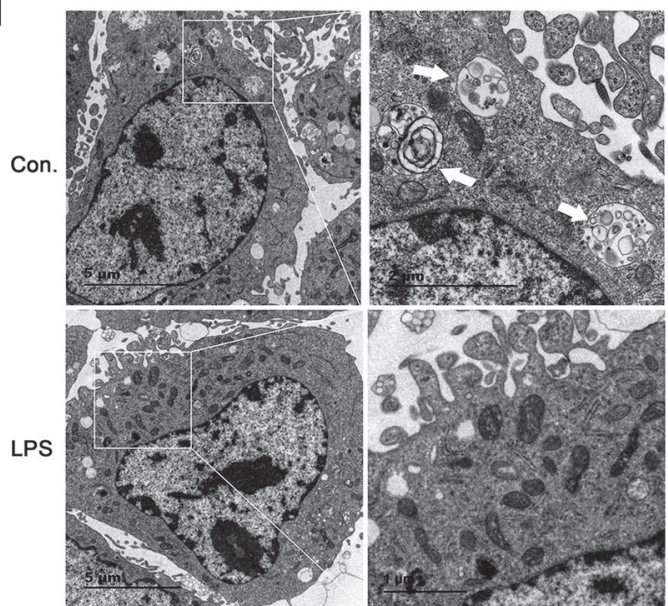

LPS challenge, as observed by fluorescence microscopy (Fig. 1c). To visually investigate this finding further, autophagosomes were detected with TEM. As shown in Fig. 1d, a number of double-membraned vesicles loaded with dysfunctional proteins or cellular debris were observed in the control group. In contrast, no autophagosomes were found in the LPS group. These findings suggest that LPS inhibits the formation of autophagosomes in MLFs and blocks autophagic flux.

\section{mTOR phosphorylation is required for LPS-inhibited autophagy}

mTOR functions as a serine/threonine protein kinase that can regulate autophagy in cellular metabolism [12]. To investigate the mechanism underlying LPS-induced autophagy inhibition, we detected the activation of mTOR in MLFs stimulated with $1 \mu \mathrm{g} / \mathrm{mL}$ LPS for $6 \mathrm{~h}$. As shown in Fig. 2a, the level of phosphorylated mTOR (p-mTOR) was relatively higher in the LPS group than in the control group, and the p-mTOR/mTOR ratio showed a statistically significant difference. These data suggest that LPS promotes the phosphorylation of mTOR.

Because mTOR inhibition is related to the induction of autophagy [13], we examined whether inhibiting the activation of mTOR physically affected autophagy in MLFs. Rapamycin is a known selective inhibitor of mTOR. As shown in Fig. 2b, pretreatment with rapamycin significantly decreased both the p-mTOR expression level and the p-mTOR/mTOR ratio. Furthermore, pretreatment with rapamycin reduced the expression of p62 (Fig. 2c), which implied that rapamycin partially rescued the autophagy 

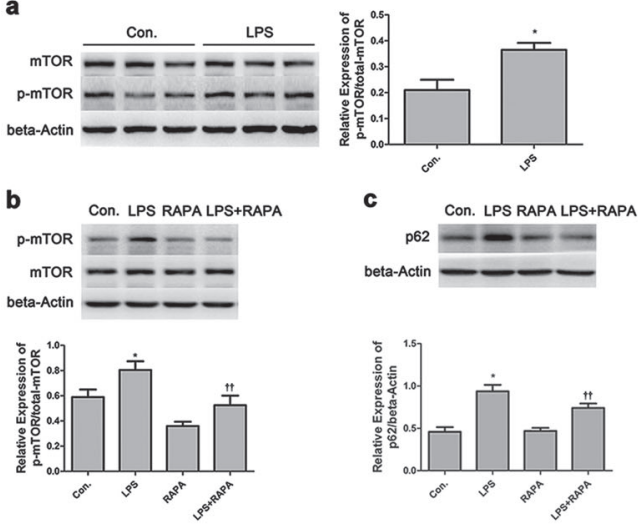

d
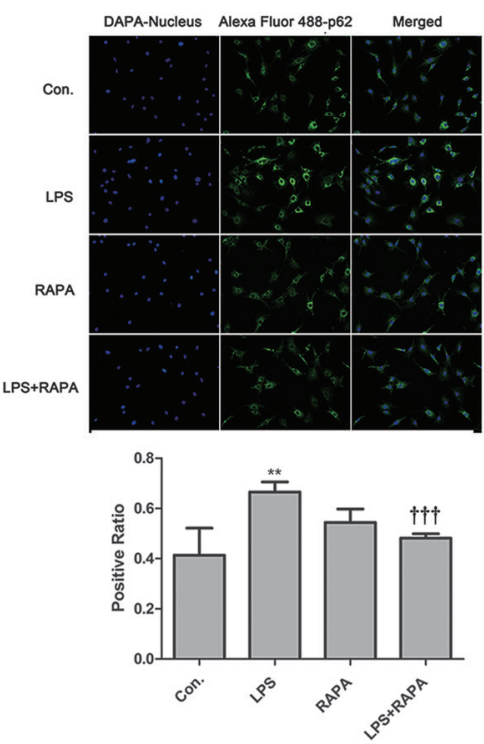

e

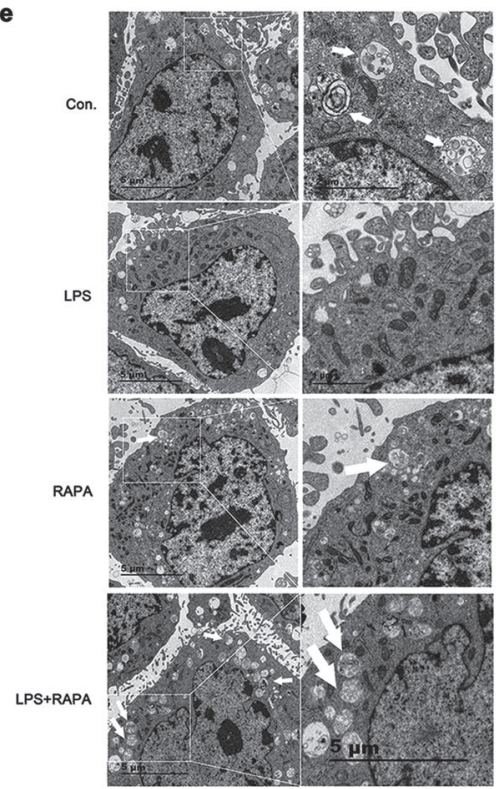

inhibited by LPS. The finding was also verified by immunofluorescence (Fig. 2d). The fluorescence intensity of p62 was higher in the LPS group than in the untreated control
Fig. 2 mTOR phosphorylation is required for LPS-inhibited autophagy. The phosphorylation of mTOR in MLFs $6 \mathrm{~h}$ after treatment with $1 \mu \mathrm{g} / \mathrm{mL}$ LPS (a) with/without mTOR pathway inhibitors (b) was detected by Western blotting. The protein expression of p62 in MLFs after being stimulated with $1 \mu \mathrm{g} / \mathrm{mL}$ LPS for $30 \mathrm{~h}$ with/without inhibitors of the mTOR pathway was detected by Western blotting (c). Rapamycin (10 nmol/L for $1 \mathrm{~h}$ prior to LPS administration) was applied to determine whether p62 expression (c, d) and autophagosome formation (e) are regulated by mTOR phosphorylation. * $p<0.05$ vs. Control group; $* * p<0.01$ vs. Control group; $\pitchfork p<0.01$ vs. LPS group; ${ }^{\dagger \dagger} p<0.001$ vs. LPS group. Columns represent mean values, and error bars represent SD. Blots are representative of three independent experiments

group, but the fluorescence intensity of the LPS + RAPA group declined and was similar to that of the control group. The TEM results also revealed that double-membrane autophagosomes reappeared in the LPS + RAPA group (Fig. 2e). These observations suggest that the phosphorylation of mTOR is required for LPS-inhibited autophagy in MLFs.

\section{Activation of the PI3K-Akt-mTOR signaling pathway is required for LPS-inhibited autophagy}

mTOR is a member of the phosphatidylinositol-3-kinaserelated kinase family of protein kinases, and our previous study verified that the PI3K-Akt pathway is required for LPS-induced lung fibroblast proliferation [9]. For these reasons, we wanted to determine whether the PI3K-Akt pathway is also involved in LPS-inhibited autophagy in MLFs. We found that LPS-stimulated MLFs had a higher expression of phosphorylated-Akt (p-Akt), suggesting that LPS promotes the activation of the PI3K-Akt signaling pathway (Fig. 3a).

Ly294002 is a selective inhibitor of the PI3K-Akt signaling pathway. As shown in Fig. $3 \mathrm{~b}$ and c, pretreatment with Ly294002 significantly inhibited the phosphorylation of Akt and partially inhibited the phosphorylation of mTOR, a downstream signaling molecule of the PI3KAkt pathway (Fig. 3c). In addition, pretreatment with Ly294002 decreased the expression of p62, suggesting that Ly294002 partially rescued the autophagy inhibited by LPS (Fig. 3d). The immunofluorescence images were consistent with the results above (Fig. 3e). The p62 fluorescence intensity was higher in the LPS group than in the untreated control group, while the fluorescence intensity of the LPS + Ly294002 group reduced to show a statistically difference with the LPS group and was similar to that of the group control. The TEM images also showed that double-membrane autophagosomes reappeared in the LPS + Ly294002 group (Fig. 3f). These findings suggest that LPS can activate the PI3K-Akt pathway and its downstream protein mTOR, thereby inhibiting autophagy in MLFs. 
Fig. 3 Activation of the PI3KAkt-mTOR signaling pathway is required for LPS-inhibited autophagy. The phosphorylation of Akt (a, b) and mTOR (c) in MLFs after being challenged for $6 \mathrm{~h}$ with $1 \mu \mathrm{g} / \mathrm{mL}$ LPS (a) with/ without PI3K-Akt pathway inhibitors $(\mathbf{b}, \mathbf{c})$ were detected by Western blotting. The protein expression of p62 in MLFs after $30 \mathrm{~h}$ of stimulation with $1 \mu \mathrm{g} / \mathrm{mL}$ LPS with/without inhibitors of the PI3K-Akt pathway was detected by Western blotting (d). Ly294002 (10 $\mu \mathrm{mol} / \mathrm{L}$ for $1 \mathrm{~h}$ prior to LPS administration) was applied to determine whether mTOR phosphorylation (c), p62 expression $(\mathbf{d}, \mathbf{e})$ and autophagosome formation (f) are regulated by $\mathrm{PI} 3 \mathrm{~K}-\mathrm{Akt}$ activation. $* p<0.05$ vs. Control group; $* * p<0.01$ vs. Control group; ${ }^{\dagger} p<0.05$ vs. LPS group; ${ }^{\dagger+} p<0.001$ vs. LPS group.

Columns represent mean values, and error bars represent SD.

Blots are representative of three independent experiments
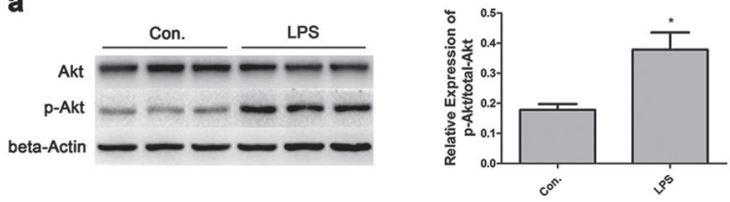
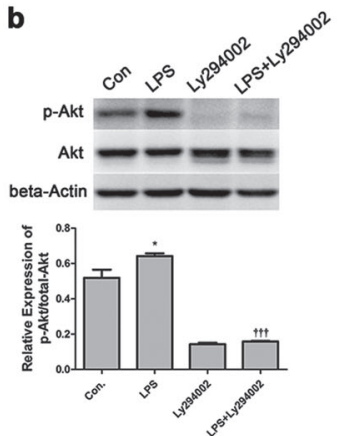

e

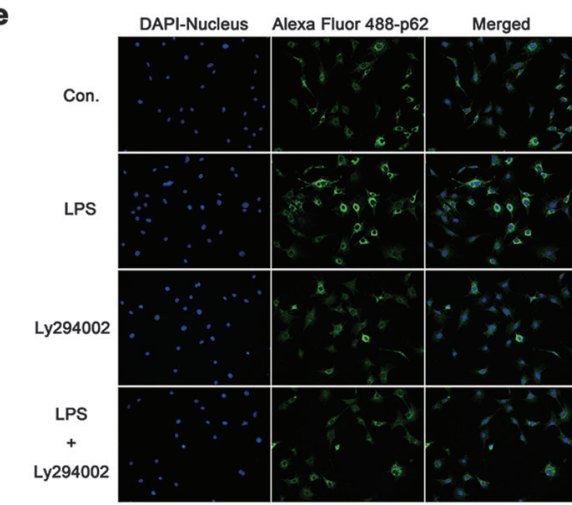

f
C $0^{2}$ d
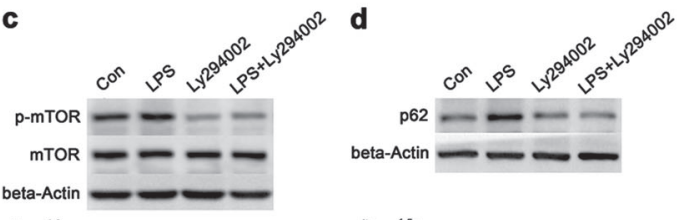
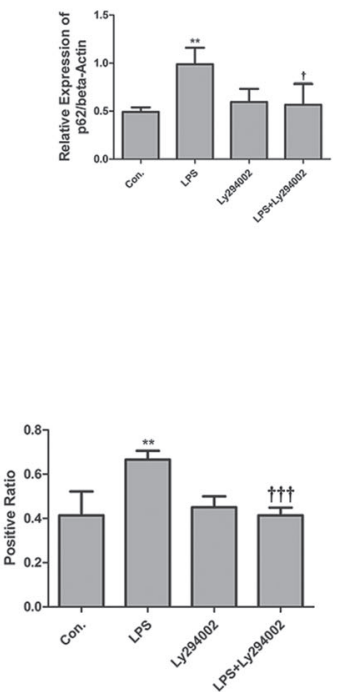

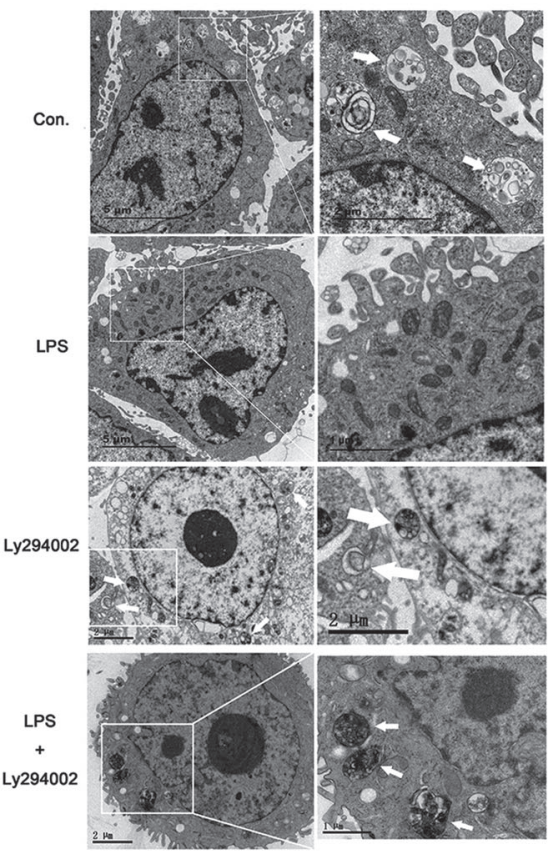


a

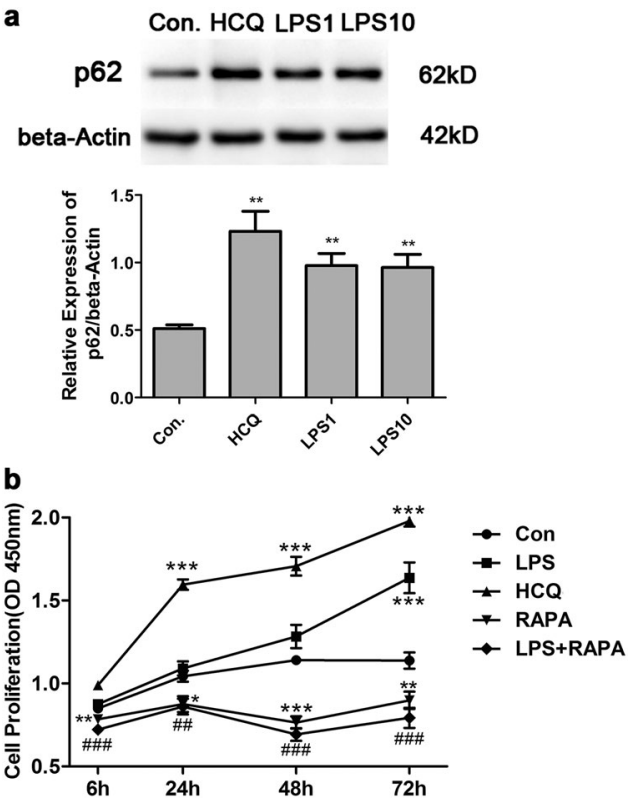

Fig. 4 Inhibiting autophagy promotes cell proliferation in mouse lung fibroblasts. The expression of p62 in mouse lung fibroblasts after being challenged for $24 \mathrm{~h}$ with $5 \mathrm{mM}$ hydroxychloroquine (HCQ) or $1 \mu \mathrm{g} / \mathrm{mL}$ LPS, $10 \mu \mathrm{g} / \mathrm{mL}$ LPS were detected by Western Blotting (a). Columns represent mean values, and error bars represent SD. Blots are representative of three independent experiments $(n=3)$. The proliferation of mouse lung fibroblasts at 6, 24, 48, $72 \mathrm{~h}$ after HCQ, LPS, rapamycin or LPS plus rapamycin challenge was detected by CCK8 assay (b). Values are expressed as mean $\pm \mathrm{SD}(n=8) .{ }^{*} p<0.05$ vs. Control group, ${ }^{* *} p<0.01$ vs. Control group, $* * * p<0.001$ vs. Control group; ${ }^{\#} p<0.01$ vs. LPS group, ${ }^{\# \#} p<0.001$ vs. LPS group

\section{Inhibiting autophagy promotes cell proliferation in MLFs}

To verify the effect of inhibiting autophagy on cell proliferation in MLFs, we use HCQ as an autophagy inhibitor in the following experiments. HCQ is a well-known antimalarial drug, and also a widely-recognized autophagy inhibitor. HCQ was administrated to MLFs with a concentration of $5 \mathrm{mM}$ according to the instruction, while LPS was applied with concentration of $1 \mu \mathrm{g} / \mathrm{mL}$ (LPS1 group) and $10 \mu \mathrm{g} / \mathrm{mL}$ (LPS10 group). The western blots results showed that the application of HCQ or LPS led to the accumulation of $\mathrm{p} 62$, indicating that autophagy was inhibited in MLFs (Fig. 4a). In the meanwhile, there's no significantly difference between LPS1 group and LPS10 group. Besides, the cell proliferation of MLFs was detected by CCK 8 assay. We found that the HCQ group showed a higher absorbance at $450 \mathrm{~nm}$ than the control group 24, 48, $72 \mathrm{~h}$ after HCQ was administrated, while LPS group showed a statistically higher absorbance $72 \mathrm{~h}$ after LPS was administrated. However, rapamycin as an autophagy inducer decreased the absorbance in RAPA group, and LPS plus rapamycin also showed a lower absorbance in LPS + RAPA group than the LPS group (Fig. 4b). Our findings manifested that inhibiting autophagy in MLFs promotes cell proliferation and the application of an autophagy inducer may suppress the cell proliferation in MLFs.

\section{Discussion}

It has been reported that the inhibition of autophagy in lung fibroblasts plays a key role in pulmonary fibrosis $[10,11]$. However, the correlation between proliferation and autophagy inhibition of lung fibroblasts and the underlying mechanism remains unknown. Our experimental results showed that LPS inhibits autophagy in MLFs through the activation of the PI3K-Akt-mTOR signaling pathway, which provides new insight into the relationship between autophagy inhibition and LPS-induced lung fibroblast proliferation during pulmonary fibrosis.

A low level of autophagy is known to exist in the lung tissues of IPF patients [10, 11]. However, it is unknown if the inhibition of autophagy promotes the development of pulmonary fibrosis and what mechanism is involved in this process. Studies have shown that LPS stimulation can indeed down-regulate autophagy through the PI3K-AktmTOR pathway in odontoblasts from neural crest-derived odontogenic mesenchymal cells [14] or in the hippocampus [15]. A recent study investigated the expression of autophagy-associated proteins in bronchoalveolar lavage (BAL) fluid in LPS-induced ARDS mice and found that autophagy was suppressed with intranasal instillations of LPS [16]. These findings are consistent with our experimental results in MLFs. Moreover, Richard SN's research showed that lung fibroblasts (LFs) cultured on collagenrich matrices demonstrate aberrant proliferation and the formation of fibroblastic foci and that a low level of autophagy in LFs leads to apoptosis resistance, which is involved in pulmonary fibrosis [10]. Yusuke Kurita's findings also showed that insufficient mitophagy enhances myofibroblast differentiation in LFs, contributing to IPF pathogenesis [17]. In addition, TGF- $\beta_{1}$-impaired autophagy has been reported as a possible mechanism for the promotion of fibrosis in IPF [18]. Although the underlying mechanism has not been clarified, these findings indicate that the inhibition of autophagy participates in IPF pathogenesis.

Our experimental results showed that LPS inhibits autophagy in MLFs via the PI3K-Akt-mTOR signaling pathway. However, little is known about LPS-inhibited autophagy in lung fibroblasts. A recent study showed that inhibiting the PI3K-Akt-mTOR signaling pathway mediated tripchlorolide-induced autophagy in lung cancer cells [19]. Additional studies have also shown that inhibited autophagy is related to the PI3K-Akt-mTOR pathway [20-22], which is consistent with our findings. Furthermore, our previous 
studies found that the PI3K-Akt signaling pathway is activated/phosphorylated in lung tissues with injury or fibrosis induced by LPS [7-9]. A recent study showed that inhibiting the PI3K-Akt-mTOR signaling pathway with different inhibitors increased the stability and expression of a critical regulator of cystic fibrosis, and the most effective inhibitor exhibited a rescue effect by restoring autophagy, showing that the inhibition of autophagy through PI3K-AktmTOR signaling pathway activation plays a critical role in cystic fibrosis [23]. In the present study, autophagy was rescued via inhibition of the PI3K-Akt-mTOR pathway with the selective inhibitors, Ly294002 and rapamycin. Therefore, we inferred that LPS inhibits autophagy in lung fibroblasts by activating the PI3K-Akt-mTOR signaling pathway, which probably plays an important role in LPS-induced pulmonary fibrosis.

Both autophagy and proliferation are cellular fundamental biological process, and researches are increasingly discovering various interactions between them. Studies showed that a low level of autophagy was detected in the lung tissues and fibroblasts of IPF patients [11], inferring a relationship between autophagy inhibition and pulmonary fibrosis. However, the correlation between proliferation and autophagy inhibition of lung fibroblasts and the underlying mechanism remains unknown. Emerging evidence has shown that activation of autophagy inhibits proliferation in tumor cells. Hu's study found that autophagy suppresses proliferation of HepG2 cells partially by inhibition of GPC3/wnt/ $\beta$-catenin signaling [24]. Furthermore, Wang's recent research in normal lung fibroblasts indicated that Elongation factor- 2 kinase might inhibit TGF- $\beta 1$-induced proliferation and differentiation and activate cell apoptosis and autophagy through p38 MAPK signaling, which might ameliorate lung fibroblast-to-myofibroblast differentiation [25]. These results also suggest an interrelationship between autophagy and proliferation that the inhibition of autophagy might promote the proliferation in lung fibroblasts, which was consistent with our findings.

In conclusion, our study provides evidence that LPS promotes lung fibroblast proliferation through autophagy inhibition via activation of the PI3K-Akt-mTOR pathway, and that intervention by targeting inhibition of lung fibroblast autophagy may be a constructive measure for treatment of pulmonary fibrosis. Data from an in vivo model would strengthen these findings, and will be considered in further studies.

Acknowledgements This research was supported by grants from the National Natural Science Foundation of China (NSFC, No. 81770060 and 81670057) and Training Program Foundation for Distinguished Young Medical Professional from Shanghai Municipal Commission of Health and Family Planning (No. 2018-16). The funders had no role in study design, data collection and analysis, decision to publish, or preparation of the manuscript.

\section{Compliance with ethical standards}

Conflict of interest The authors declare that they have no conflict of interest.

Publisher's note: Springer Nature remains neutral with regard to jurisdictional claims in published maps and institutional affiliations.

\section{References}

1. Marshall RP, Bellingan G, Webb S, Puddicombe A, Goldsack N, McAnulty RJ et al. Fibroproliferation occurs early in the acute respiratory distress syndrome and impacts on outcome. Am J Respir Crit Care Med. 2000;162:1783-8.

2. Marshall R, Bellingan G, Laurent G. The acute respiratory distress syndrome: fibrosis in the fast lane. Thorax. 1998;53:815-7.

3. He Z, Wang X, Deng Y, Li W, Chen Y, Xing S et al. Epigenetic regulation of Thy-1 gene expression by histone modification is involved in lipopolysaccharide-induced lung fibroblast proliferation. J Cell Mol Med. 2013;17:160-7.

4. Li W, Xu QY, Deng YX, Yang ZW, Xing SP, Zhao XY et al. High-mobility group box 1 accelerates lipopolysaccharideinduced lung fibroblast proliferation in vitro: involvement of the NF-kappa B signaling pathway. Lab Invest. 2015;95:635-47.

5. S D, H L, Y C, L Y, J W, H H et al. Houttuynia cordata inhibits lipopolysaccharide-induced rapid pulmonary fibrosis by upregulating IFN- $\gamma$ and inhibiting the TGF- $\beta 1 /$ Smad pathway. Int Immunopharmacol. 2012;13:331-40.

6. Li H, Du S, Yang L, Chen Y, Huang W, Zhang R et al. Rapid pulmonary fibrosis induced by acute lung injury via a lipopolysaccharide three-hit regimen. Innate Immun. 2009;15:143-54.

7. $\mathrm{He} \mathrm{Z}$, Zhu Y, Jiang $\mathrm{H}$. Toll-like receptor 4 mediates lipopolysaccharide-induced collagen secretion by phosphoinositide3kinase-Akt pathway in fibroblasts during acute lung injury. J Recept Signal Transduct Res. 2009;29:119-25.

8. He Z, Deng Y, Li W, Chen Y, Xing S, Zhao X et al. Overexpression of PTEN suppresses lipopolysaccharide-induced lung fibroblast proliferation, differentiation and collagen secretion through inhibition of the PI3-K-Akt-GSK3beta pathway. Cell Biosci. 2014;4:2

9. He ZY, Gao Y, Deng YX, Li W, Chen YM, Xing SP et al. Lipopolysaccharide Induces Lung Fibroblast Proliferation through Toll-Like Receptor 4 Signaling and the Phosphoinositide3Kinase-Akt Pathway. PLoS ONE. 2012;7:e35926.

10. Nho RS, Hergert P. IPF fibroblasts are desensitized to type I collagen matrix-induced cell death by suppressing low autophagy via aberrant Akt/mTOR kinases. PLoS ONE. 2014;9: e94616.

11. Jintaek I, Polla H, Richard Seonghun N. Reduced FoxO3a expression causes low autophagy in idiopathic pulmonary fibrosis fibroblasts on collagen matrices. Am J Physiol Lung Cell Mol Physiol. 2015;309:552-61.

12. Switon K, Kotulska K, Janusz-Kaminska A, Zmorzynska J, Jaworski J. Molecular neurobiology of mTOR. Neuroscience. 2017;341:112-53.

13. Wu Y, Li Y, Zhang H, Huang Y, Zhao P, Tang Y et al. Autophagy and mTORC1 regulate the stochastic phase of somatic cell reprogramming. Nat Cell Biol. 2015;17:715-25.

14. Pei F, Lin H, Liu H, Li L, Zhang L, Chen Z. Dual role of autophagy in lipopolysaccharide-induced preodontoblastic cells. J Dent Res. 2015;94:175-82.

15. Francois A, Terro F, Quellard N, Fernandez B, Chassaing D, Janet $\mathrm{T}$ et al. Impairment of autophagy in the central nervous system 
during lipopolysaccharide-induced inflammatory stress in mice. Mol Brain. 2014;7:56.

16. Yang X, Jing T, Li Y, He Y, Zhang W, Wang B et al. Hydroxytyrosol attenuates LPS-induced acute lung injury in mice by regulating autophagy and sirtuin expression. Curr Mol Med. 2017;17:149-59.

17. Kurita Y, Araya J, Minagawa S, Hara H, Ichikawa A, Saito N et al. Pirfenidone inhibits myofibroblast differentiation and lung fibrosis development during insufficient mitophagy. Respir Res. 2017;18:114.

18. Patel AS, Lin L, Geyer A, Haspel JA, An CH, Cao J et al. Autophagy in idiopathic pulmonary fibrosis. PLoS ONE. 2012;7:e41394.

19. Chen LM, Song TJ, Xiao JH, Huang ZH, Li Y,Lin TY. Tripchlorolide induces autophagy in lung cancer cells by inhibiting the $\mathrm{PI} 3 \mathrm{~K} / \mathrm{AKT} / \mathrm{mTOR}$ pathway and improves cisplatin sensitivity in A549/DDP cells. Oncotarget. 2017;8:63911-22.

20. Guan H, Piao H, Qian ZQ, Zhou XY, Sun YJ, Gao CX et al. 2,5Hexanedione induces autophagic death of VSC4.1 cells via a PI3K/Akt/mTOR pathway. Mol Biosyst. 2017;13:1993-2005.
21. Du C, Zhang T, Xiao X, Shi Y, Duan H, Ren Y. Protease-activated receptor-2 promotes kidney tubular epithelial inflammation by inhibiting autophagy via the PI3K/Akt/mTOR signalling pathway. Biochem J. 2017;474:2733-47.

22. Zhong JT, Yu J, Wang HJ, Shi Y, Zhao TS, He BX et al. Effects of endoplasmic reticulum stress on the autophagy, apoptosis, and chemotherapy resistance of human breast cancer cells by regulating the PI3K/AKT/mTOR signaling pathway. Tumour Biol. 2017;39:1010428317697562.

23. Reilly R, Mroz MS, Dempsey E, Wynne K, Keely SJ, McKone $\mathrm{EF}$ et al. Targeting the PI3K/Akt/mTOR signalling pathway in Cystic Fibrosis. Sci Rep. 2017;7:7642.

24. Hu P, Cheng B, He Y, Wei Z, Wu D, Meng Z. Autophagy suppresses proliferation of HepG2 cells via inhibiting glypican-3/wnt/ $\beta$-catenin signaling. Oncotargets Ther. 2018;11:193.

25. Wang Y, Huang G, Wang Z, Qin H, Mo B, Wang C. Elongation factor-2 kinase acts downstream of p38 MAPK to regulate proliferation, apoptosis and autophagy in human lung fibroblasts. Exp Cell Res. 2018;363:291-8. 